\title{
Photoluminescence Dynamics in Singlet Fission Chromophore Liquid Melts
}

\author{
Geoffrey B. Piland and Christopher J. Bardeen* \\ Department of Chemistry \\ University of California, Riverside \\ Riverside, CA 92506 \\ *christopher.bardeen@ucr.edu
}

\begin{abstract}
$\underline{\text { Abstract }}$
The effect of high temperature melting on the photophysics of three prototypical singlet fission molecules is investigated. Time-resolved photoluminescence is used to look at the melt phase of the molecules tetracene, diphenylhexatriene and rubrene. Chemical decomposition of tetracene precluded any detailed measurements on this molecule. In the diphenylhexatriene melt, a rapid singlet state nonradiative relaxation process outcompetes singlet fission. In the rubrene melt, singlet fission occurs at a rate similar to that of the crystal, but the decay of the delayed fluorescence is much more rapid. The rapid decay of the delayed fluorescence suggests that either the triplet lifetime is shortened, or the fusion probability decreases, or that both factors are operative at higher temperatures.
\end{abstract}

(C) 2016. This manuscript version is made available under the Elsevier user license http://www.elsevier.com/open-access/userlicense/1.0/ 


\section{$\underline{\text { Introduction }}$}

Interest in singlet fission (SF) as a route toward more efficient solar energy conversion has inspired considerable theoretical and experimental work in order to understand how its rate depends on molecular packing. ${ }^{1-2}$ Perhaps the simplest way to experimentally vary intermolecular interactions is to go from the ordered environment of a crystal to a disordered environment. Recent results on pentacene and tetracene derivatives in statically disordered amorphous films $^{3-7}$ and dynamically disordered liquids ${ }^{8-11}$ have demonstrated that crystalline order between separate chromophores is not a requirement for rapid SF.

One disadvantage of disordered solids is that triplet exciton diffusion tends to be much slower than in a single crystal. This is because of the existence of static, low-energy sites that trap the exciton, preventing it from hopping to neighboring sites. However, simulations by Bässler suggested that disorder is not necessarily fatal for triplet exciton diffusion in a fluid environment. $^{12}$ Experiments on molten anthracene and naphthalene showed that these liquids could support long triplet lifetimes and enhanced triplet diffusion lengths. ${ }^{13-15}$ Later theoretical $^{16}$ and experimental ${ }^{17-18}$ work on energy migration in dye molecule solutions demonstrated that liquid environments could also enhance singlet exciton diffusion rates. Presumably, the ability of liquid-state molecules to sample multiple configurations during their excited state lifetimes prevents the formation of static low energy trap sites. Since SF is already known to occur in disordered environments, we became interested in whether melt-phase SF chromophores could exhibit both SF and the long triplet lifetimes seen in anthracene melts.

In this work, we used time-resolved photoluminescence (PL) to look at the melt phase of the prototypical SF molecules rubrene (RUB), tetracene (TET) and diphenylhexatriene (DPH). We were hoping to find evidence that rapid SF was retained, as well as long triplet lifetimes as 
observed for anthracene. Such a result would provide evidence that SF in a fluid medium could be a viable approach to making largescale homogeneous layers. The bulk of this paper will concentrate on RUB, where SF occurs in the melt but the triplet dynamics change considerably. In DPH, a new singlet relaxation channel arises that appears to outcompete SF. We could not measure reproducible data for TET due to chemical decomposition in the melt. In addition to being the first characterization of PL lifetimes for the melt phase of several important organic semiconductors, this work also suggests that chemical decomposition and accelerated nonradiative decay rates in melts can make them less than ideal phases for SF.

\section{$\underline{\text { Experimental }}$}

Rubrene samples were prepared by placing 99.9\% rubrene from Acros Organics into a melting point capillary until the level reached halfway up the tube. The capillary was evacuated until the internal pressure reached 1 mTorr. While still pulling vacuum on the sample, the capillary was flame sealed, leaving an ample space at the top to insure the flame did not directly heat the sample.

For high-temperature spectroscopic measurements, the sealed samples were placed within a modified Laboratory Devices Mel-Temp melting point apparatus. The faceplate of the melting point apparatus was removed and a custom aluminum faceplate was constructed to allow for excitation and photoluminescence collection. Temperatures of the melting point apparatus were measured using a Digi-Sense Thermocouple Thermometer. Magnetic field effects were tested through the use of a pair of cylindrical magnets (K\&J Magnetics, part number DY0Y0-N50) that were positioned on both sides of the melting point apparatus. A Gauss-meter was used to measure a $1.5 \mathrm{kG}$ magnetic field strength at the sample position. 
Time-resolved PL data were obtained using front-face detection with a Hamamatsu C4334 Streakscope picosecond streak camera with $2.5 \mathrm{~nm}$ wavelength resolution and $15 \mathrm{ps}$ time resolution. Unless otherwise specified, the fluorescence decays were integrated over 550-700 nm. The $800 \mathrm{~nm}$ output of a Coherent Libra regenerative amplifier rate (1 KHz repetition rate) was frequency doubled in a beta-barium borate crystal to generate the $400 \mathrm{~nm}$ pulses, which were then focused with a $20 \mathrm{~cm}$ focal length lens onto the samples in the capillary.

\section{$\underline{\text { Results and Discussion }}$}

The PL spectra of crystalline RUB can vary considerably. In general, there is a high energy emission with a well-defined vibronic peak progression, starting at $560 \mathrm{~nm}$, often accompanied by a red-shifted emission that is centered from $620 \mathrm{~nm}$ to $650 \mathrm{~nm}$ and is more or less featureless. ${ }^{19}$ The $560 \mathrm{~nm}$ emission is intrinsic to the crystal, while the redshifted emission was originally assigned to oxidized rubrene molecules ${ }^{20}$ but is now thought to originate from disordered regions of the crystal (these may be generated by oxidation of surrounding molecules). ${ }^{21-22}$ The steady-state PL of crystalline samples is usually a mixture of both types. Our samples were powdered in order to pack the melting point capillary, and this process leads to an unstructured emission peaked at around $600 \mathrm{~nm}$, shown in Figure 1, the same wavelength observed for most single crystals. ${ }^{21,23}$ This redshifted emission probably reflects strong selfabsorption in the sample ${ }^{19}$, but may also include contributions from defect states created by powdering. After the crystal melts, this emission shifts slightly to higher energy and broadens, but does not undergo dramatic changes in shape or intensity. After the sample is cooled down again, we find that the emission of the solid has redshifted to $650 \mathrm{~nm}$. This is consistent with the findings of Podzorov and coworkers that melting and reforming the crystal leads to enhanced 
low-energy emission. ${ }^{21}$ This melt-induced change in the PL is not due to chemical decomposition. When the melted crystal was redissolved in methylene chloride, the original RUB absorption and fluorescence spectra were recovered.

In crystalline form, the RUB PL signal reflects both SF and triplet fusion, which occur on different timescales. ${ }^{24-26}$ These two processes lead to a prompt PL decay that reflects the lifetime of the initially excited singlet state, followed by a longer-lived component due to delayed fluorescence generated by triplet-triplet recombination. In the first $100 \mathrm{ps,} \mathrm{where} \mathrm{the}$ signal decays by about one natural log, the decay is dominated by the $S_{1} \rightarrow T_{1}+T_{1}$ fission process. The prompt decay in the $1 \mathrm{~ns}$ time window, shown in Figure 2a, corresponds to a singlet lifetime of $\sim 60$ ps that remains remarkably constant over the temperature range $20-340^{\circ} \mathrm{C}$. Femtosecond transient absorption experiments on crystalline RUB suggested that the majority of SF occurs within $1 \mathrm{ps}^{27}$, but later experiments indicated that this rate may depend on excitation density and thermal load. ${ }^{23}$ Our excitation densities are below those typically used in transient absorption experiments, and our SF time of 60 ps approaches the 35 ps time seen at the lowest fluences in such experiments. ${ }^{23}$ But the distorted PL spectrum makes it impossible to unequivocally identify the emitting species as the high energy species, so it is also possible that we are measuring the dynamics of a subset of sites within the crystal rather than the majority species seen in the transient absorption experiments.

After $100 \mathrm{ps}$, the $\mathrm{T}_{1}+\mathrm{T}_{1} \rightarrow \mathrm{S}_{1}$ fusion processes start to equilibrate the singlet and triplet populations. Up to $310^{\circ} \mathrm{C}$, the post-100 ps region of the PL decay is unchanged to within the experimental noise. After melting at $340^{\circ} \mathrm{C}$, there is a noticeable slowing of the later decay in the $0.1-0.8 \mathrm{~ns}$ region. This slight increase in the PL signal may reflect an increased fusion rate, rather than slower fission. When we shift to observing the PL decay on longer timescales, the 
delayed fluorescence decreases dramatically, as shown in Figure 2b. The PL spectra in both the early and late time regimes are identical (Figure 2b, inset), confirming that the long decay component reflects delayed fluorescence. Note that the slight enhancement of the melt PL in the $1 \mathrm{~ns}$ window is canceled out by a more rapid PL decay over the next $20 \mathrm{~ns}$. The crossing of the melt and $20^{\circ} \mathrm{C}$ decays can be discerned in Figure $2 \mathrm{a}$ at about $0.8 \mathrm{~ns}$.

To confirm that the PL decay reflects SF dynamics, we measure it in the absence and presence of a magnetic field. As observed in solid amorphous films ${ }^{4}$ and crystals ${ }^{24}$, the RUB samples exhibit a strong magnetic field enhancement of the fluorescence on intermediate to long timescales in both the room temperature solid and in the melt (Figure 3). In an earlier paper, we calculated that enhanced fluorescence in the presence of a strong magnetic field was a symptom of aligned pairs. ${ }^{4}$ However, recent work by Tapping and Huang showed our analysis was flawed and that such an enhancement occurs for both aligned and randomly oriented pairs. ${ }^{28}$ So the positive sign of the magnetic field effect is consistent with SF occurring between randomly oriented molecular pairs, as expected in a liquid. The magnetic field effect supports our hypothesis that the prompt and delayed PL decays reflect fission and fusion dynamics, respectively.

Because we are focusing into a $1 \mathrm{~mm}$ diameter capillary, a precise calculation of excitation density in the sample was not possible. Nevertheless, we did vary the incident excitation pulse intensity over an order of magnitude (1-15 microwatts, before focusing) and observed no change in the PL decay of the RUB melt, similar to our results on solid amorphous RUB. ${ }^{4}$ The lack of an intensity dependence for the delayed fluorescence is consistent with the dominant role of geminate recombination in our experimental regime. If nongeminate recombination played a significant role, this contribution would depend quadratically on 
excitation density and the amplitude of the delayed fluorescence relative to the prompt fluorescence would increase with laser intensity.

The rapid decay of RUB's delayed fluorescence at elevated temperatures could arise from several different physical mechanisms. One concern we had was that rapid diffusion of small impurities like $\mathrm{O}_{2}$ or metal atoms might lead to PL quenching in the high temperature melt. We tested for such effects in two ways. First, we compared results from two different RUB samples: one from crystals used as received from Acros Organics, and one from a ground-up single crystal grown by gradient vapor phase deposition, which presumably would have a lower level of chemical impurities. Both samples gave similar PL decays upon melting. Second, we tested for the effect of residual $\mathrm{O}_{2}$ by evacuating a capillary to $10^{-3}$ Torr before flame sealing, and compared the results to those from a capillary that was not evacuated. Again, the PL signals from the different preparations were the same to within a few percent. Thus we think that the photophysical changes observed upon melting reflect changes in the intrinsic material structure, rather than changes in extrinsic impurity quenching.

To better understand the origin of the delayed fluorescence decay, we consider the simple kinetic model outlined in Equations (1) and (2). ${ }^{4}$ We assume that the triplet population consists of associated pairs (the $T T$ state) produced by fission that can fusion back into the singlet state. This leads to a simple pair of rate equations:

$$
\begin{aligned}
& \frac{d N_{S 1}}{d t}=-k_{f i s s} N_{S 1}+k_{f u s} N_{T T} \\
& \frac{d N_{T T}}{d t}=-k_{t r i p} N_{T T}-k_{f u s} N_{T T}+k_{f i s s} N_{S 1}
\end{aligned}
$$

where $k_{f i s s}$ is the fission rate, $k_{f u s}$ is the fusion rate, and $k_{\text {trip }}$ is the triplet decay rate. Two factors will affect whether the triplets produced by SF can give rise to delayed fluorescence: their 
ability to fuse, as given by $k_{f u s}$, and their lifetime, as determined by $k_{t r i p}$. In Figure 4 , we show calculated PL decays obtained by allowing either $k_{f u s}$ to decrease or $k_{\text {trip }}$ to increase with temperature. In both cases, the delayed fluorescence signal decreases. Comparing the calculated curves in Figure 4 to the experimental decays in Figure 2b, we see elements of both a decreased amplitude (predicted by a decreased $k_{f u s}$ ) and a more rapid decay (as predicted by an increased $\left.k_{\text {trip }}\right)$. Both effects are expected. The fluid environment of the melt may facilitate triplet energy migration and separation of triplet pairs, lowering the probability of geminate fusion and thus $k_{f u s}$, as shown in Figure 4a. More rapid triplet energy migration would also increase the probability of nongeminate fusion, but the lack of intensity dependence described in the preceding paragraph suggests that our excitation densities are low enough that this process is negligible. The existence of an intramolecular decay process for rubrene's triplet state has been inferred from solution measurements ${ }^{29}$, and the activation energy from that work predicts a factor of 50 increase in $k_{\text {trip }}$ from $298^{\circ} \mathrm{C}$ to $340^{\circ} \mathrm{C}$. This is the same order of magnitude of the change seen in the simulations in Figure $4 \mathrm{~b}$. A more quantitative analysis of the relative roles of $k_{f u s}$ and $k_{\text {trip }}$ would require more sophisticated modeling. Equations (1) and (2) do not include phenomena, like diffusion ${ }^{30-32}$, that would be needed to accurately model the full dynamics of the triplet pairs.

Finally, we wanted to see if the changes in the RUB melt could be observed in other SF molecules. Our attempts to measure the PL of the TET melt were not successful due to chemical decomposition at elevated temperatures in the melt. After cooling, the resultant solid had turned from orange to black and could not be fully dissolved in methylene chloride. The origin of this decomposition is not clear - it may involve residual impurities. $\mathrm{O}_{2}$ is a possible candidate, but the dark color and insolubility of the solid make endoperoxide formation unlikely, as this product 
would be soluble and absorb in the ultraviolet. ${ }^{33}$ During these experiments, we did observe a single exponential decay for vapor phase tetracene with a lifetime of about 4 ns at $370{ }^{\circ} \mathrm{C}$.

DPH did not decompose during the melting experiments, and its time-resolved PL data are shown in Figure 5. At room temperature, in a $1 \mathrm{~ns}$ window, the PL decay of the DPH sample is slightly slower than in our previous paper. ${ }^{34}$ This may be due to having a mixture of crystal polymorphs in this powdered sample, since the SF rate is sensitive to molecular packing. Unlike RUB, both the prompt and delayed components are strongly affected by melting. The prompt decay time decreases from 130 ps to 20 ps in the melt, and the delayed fluorescence signal disappears completely. There was no measurable magnetic field effect on the PL decay in the melt, in contrast to crystalline DPH at room temperature. ${ }^{34}$ We conclude that melting gives rise to a rapid nonradiative relaxation that competes effectively with SF. This lifetime shortening is not simply due to increased conformational freedom in the melt, because in room temperature liquid solutions DPH has an excited state lifetime on the order of nanoseconds. ${ }^{35}$ Saltiel has suggested there exist intramolecular conversion pathways for DPH that do not involve torsional motion $^{36}$, and if one of these pathways is thermally activated, it could explain the rapid relaxation in the high-temperature melt. As with RUB, we did not observe any measurable change in the steady state absorption or fluorescence after the melted crystal was redissolved in methylene chloride, suggesting that chemical decomposition was negligible in this sample.

\section{Conclusion}

In this paper, we examined how high temperature melting affects the photophysics of three prototypical SF molecules. Chemical decomposition of TET prevented us from making any concrete observations on this molecule. In the DPH melt, a rapid singlet state nonradiative 
relaxation short-circuits SF altogether. RUB is the most interesting case, because in the melt it undergoes SF at a similar rate to that observed for the room temperature crystalline powder. The ensuing triplet dynamics are quite different, however, with a rapid decay of the delayed fluorescence that suggests the triplet lifetime is shortened or possibly pair dissociation is enhanced. Transient absorption experiments would help clarify which factor determines the fate of the triplets at high temperatures. It is possible that a liquid composed of RUB chromophores (e.g. a derivative prepared as ionic liquid) might exhibit the desired combination of rapid SF and long triplet lifetimes and diffusion lengths.

\section{$\underline{\text { Acknowledgements }}$}

This work was supported by the National Science Foundation under grant DMR-1508099. 


\section{$\underline{\text { References }}$}

(1) Smith, M. B.; Michl, J., Recent advances in singlet fission. Ann. Rev. Phys. Chem. 2013, 64, 361-386.

(2) Piland, G. B.; Burdett, J. J.; Dillon, R. J.; Bardeen, C. J., Singlet Fission: From Coherences to Kinetics. J. Phys. Chem. Lett. 2014, 5, 2312-2319.

(3) Roberts, S. T.; McAnally, E. R.; Mastron, J. N.; Webber, D. H.; Whited, M. T.; Brutchey, R. L.; Thompson, M. E.; Bradforth, S. E., Efficient singlet fission discovered in a disordered acene film. J. Am. Chem. Soc. 2012, 134, 6388-6400.

(4) Piland, G. B.; Burdett, J. J.; Kurunthu, D.; Bardeen, C. J., Magnetic field effects on singlet fission and fluorescence decay dynamics in amorphous rubrene. J. Phys. Chem. C 2013, $117,1224-1236$.

(5) Jankus, V.; Snedden, E. W.; Bright, D. W.; Arac, E.; Dai, D.; Monkman, A. P., Competition Between Polaron Pair Formation and Singlet Fission Observed in Amorphous Rubrene Films. Phys. Rev. B 2013, 87, 224202/1-7.

(6) Pensack, R. D.; Tilley, A. J.; Parkin, S. R.; Lee, T. S.; Payne, M. M.; Gao, D.; Jahnke, A. A.; Oblinsky, D. G.; Li, P.-F.; Anthony, J. E.; Seferos, D. S.; Scholes, G. D., Exciton Delocalization Drives Rapid Singlet Fission in Nanoparticles of Acene Derivatives. J. Am. Chem. Soc. 2015, 137, 6790-6803.

(7) Tayebjee, M. J. Y.; Schwarz, K. N.; MacQueen, R. W.; Dvořák, M.; Lam, A. W. C.; Ghiggino, K. P.; McCamey, D. R.; Schmidt, T. W.; Conibeer, G. J., Morphological Evolution and Singlet Fission in Aqueous Suspensions of TIPS-Pentacene Nanoparticles. J. Phys. Chem. C 2016, 120, 157-165.

(8) Walker, B. J.; Musser, A. J.; Beljonne, D.; Friend, R. H., Singlet Exciton Fission in Solution. Nat. Chem. 2013, 5, 1019-1024.

(9) Stern, H. L.; Musser, A. J.; Gelinas, S.; Parkinson, P.; Herz, L. M.; Bruzek, M. J.; Anthony, J.; Friend, R. H.; Walker, B. J., Identification of a Triplet Pair Intermediate in Singlet Exciton Fission in Solution. Proc. Nat. Acad. Sci. 2015, 112, 7656-7661.

(10) Thompson, N. J.; Hontz, E.; Chang, W.; Voorhis, T. V.; Baldo, M., Magnetic Field Dependence of Singlet Fission in Solutions of Diphenyl tetracene. Phil. Trans. R. Soc. A 2015, 373, 20140323/1-7.

(11) Zhang, Y.-D.; Wu, Y.; Xu, Y.; Wang, Q.; Liu, K.; Chen, J.-W.; Cao, J.-J.; Zhang, C.; Fu, H.; Zhang, H.-L., Excessive Exoergicity Reduces Singlet Exciton Fission Efficiency of Heteroacenes in Solutions. J. Am. Chem. Soc. 2016, 138, 6739-6745.

(12) Pautmeier, L.; Ries, B.; Richert, R.; Bassler, H., Disorder-Enhanced Triplet Exciton Diffusion in Condensed Aromatic Systems. Chem. Phys. Lett. 1988, 143, 459-462.

(13) Baessler, H.; Loelkes, G.; Vaubel, G., Lifetime and Motion of Triplet States in Molten Aromatic Systems. J. Chem. Phys. 1969, 51, 3695-3701.

(14) Holzman, P.; Jarnagin, R. C., Triplet Energy Migration in Pure Fluids. J. Chem. Phys. 1969, 51, 2251-2253.

(15) Kellogg, R. B.; Prock, A., Triplet Diffusion and Energy Eransfer in Molten Naphthalene. J. Chem. Phys. 1975, 63, 3161-3167.

(16) Jang, S.; Shin, K. J.; Lee, S., Effects of excitation migration and translational diffusion in the luminescence quenching dynamics. J. Chem. Phys. 1995, 102, 815-827.

(17) Joshi, H. C.; Mishra, H.; Tripathi, H. B.; Pant, T. C., Role of diffusion in excitation energy transfer: a time-resolved study. J. Luminescence 2000, 90, 17-25. 
(18) Colby, K. A.; Bardeen, C. J., Electronic Energy Migration in Solid versus Liquid Host Matrices for Concentrated Perylenediimide Dye Solutions. J. Phys. Chem. A 2011, 115, 75747581.

(19) Irkhin, P.; Ryasnyanskiy, A.; Koehler, M.; Biaggio, I., Absorption and photoluminescence spectroscpy of rubrene single crystals. Phys. Rev. B 2012, 86, 085143/1$085143 / 13$.

(20) Mitrofanov, O.; Lang, D. V.; Kloc, C.; Wikberg, J. M.; Siegrist, T.; So, W.-Y.; Sergent, M. A.; Ramirez, A. P., Oxygen-Related Band Gap State in Single Crystal Rubrene. Phys. Rev. Lett. 2006, 97, 166601/1-4.

(21) Chen, Y.; Lee, B.; Fu, D.; Podzorov, V., The Origin of a 650 nm Photoluminescence Band in Rubrene. Adv. Mater. 2011, 23, 5370-5375.

(22) Mastrogiovanni, D. D. T.; Mayer, J.; Wan, A. S.; Vishnyakov, A.; Neimark, A. V.; Podzorov, V.; Feldman, L. C.; Garfunkel, E., Oxygen Incorporation in Rubrene Single Crystals. Sci. Rep. 2014, 4, 4753/1-6.

(23) Ishibashi, Y.; Inoue, Y.; Asahi, T., The Excitation Intensity Dependence of Singlet Fission Dynamics of a Rubrene Microcrystal Studied by Femtosecond Transient Microspectroscopy. Photochem. Photobio. Sci. 2016, online.

(24) Rumyantsev, B. M.; Lesin, V. I.; Frankevich, E. L., Fluorescence of Crystalline Rubrene in a Magnetic Field. Opt. Spect. 1975, 38, 49-50.

(25) Tarasov, V. V.; Zorianiants, G. E.; Shushin, A. I.; Triebel, M. M., The role of spin-lattice relaxation in magnetic field effects on the luminescence of amorphous and polycrystalline rubrene films. Chem. Phys. Lett. 1997, 267, 58-64.

(26) Biaggio, I.; Irkhin, P., Extremely Efficient Exciton Fission and Fusion and its Dominant Contribution to the Photoluminescence Yield in Rubrene Single Crystals. Appl. Phys. Lett. 2013, 103, 263301/1-4.

(27) Ma, L.; Zhang, K.; Kloc, C.; Sun, H.; Michel-Beyerle, M. E.; Gurzadyan, G. G., Singlet fission in rubrene single crystal: direct observation by femtosecond pump-probe spectroscopy. Phys. Chem. Chem. Phys. 2012, 14, 8307-8312.

(28) Tapping, P. C.; Huang, D. M., Comment on "Magnetic Field Effects on Singlet Fission and Fluorescence Decay Dynamics in Amorphous Rubrene". J. Phys. Chem. C 2016, ASAP.

(29) Itoh, K.; Honda, K., Sensitized Delayed Fluorescence of Rubrene. Large Temperature Dependence of Triplet Lifetime of Rubrene. Chem. Phys. Lett. 1982, 87, 213-216.

(30) Najafov, H.; Lee, B.; Zhou, Q.; Feldman, L. C.; Podzorov, V., Observation of long-range exciton diffusion in highly ordered organic semiconductors. Nat. Mater. 2010, 9, 938-943.

(31) Irkhin, P.; Biaggio, I., Direct imaging of anisotropic exciton diffusion and triplet diffusion length in rubrene single crystals. Phys. Rev. Lett. 2011, 107, 017402/1-017402/4.

(32) Ryansnyanskiy, A.; Biaggio, I., Triplet exciton dynamics in rubrene single crystals. Phys. Rev. B 2011, 84, 193203/1-193203/4.

(33) Bjarneson, D. W.; Petersen, N. O., The Photochemistry of Naphthacene in Solution. $J$. Photochem. Photobiol. A 1992, 63, 327-335.

(34) Dillon, R. J.; Piland, G. B.; Bardeen, C. J., Different rates of singlet fission in monoclinic versus orthorhombic crystal forms of diphenylhexatriene. J. Am. Chem. Soc. 2013, 135, 1727817281.

(35) Cehelnik, E. D.; Cundall, R. B.; Lockwood, J. R.; Palmer, T. F., Solvent and Temperature Effects on the Fluorescence of all-trans-1 ,6-DiphenyI-1,3,5-hexatriene. J. Phys. Chem. 1975, 79, 1369-1376. 
(36) Saltiel, J.; Krishnamoorthy, G.; Huang, Z.; Ko, D.-H.; Wang, S., Photoisomerization of all-trans-1,6-Diphenyl-1,3,5-hexatriene. Temperature and Deuterium Isotope Effects. $J$. Phys. Chem. A 2003, 107, 3178-3186. 


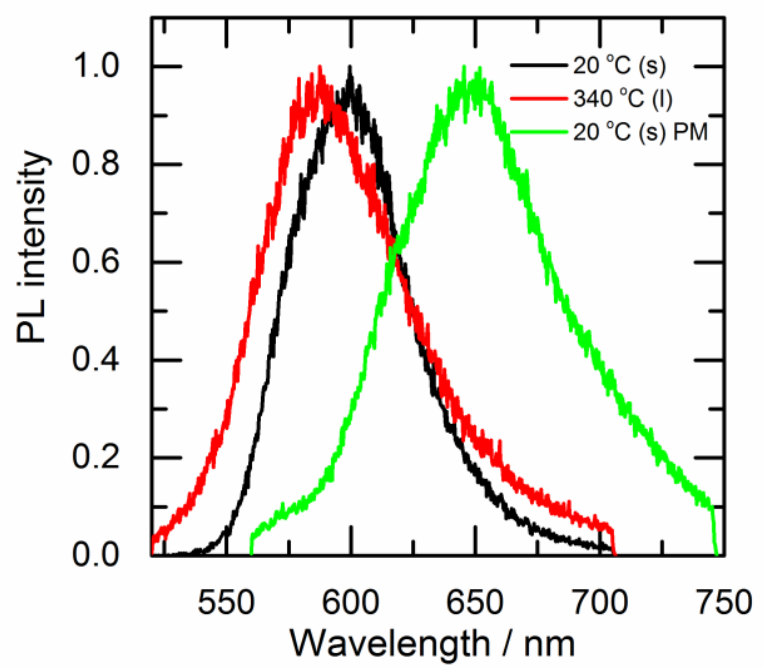

Figure 1. Time-integrated photoluminescence spectra of solid rubrene powder at room temperature (black), after melting at $340{ }^{\circ} \mathrm{C}$ (red) and after cooling back to room temperature (green). 

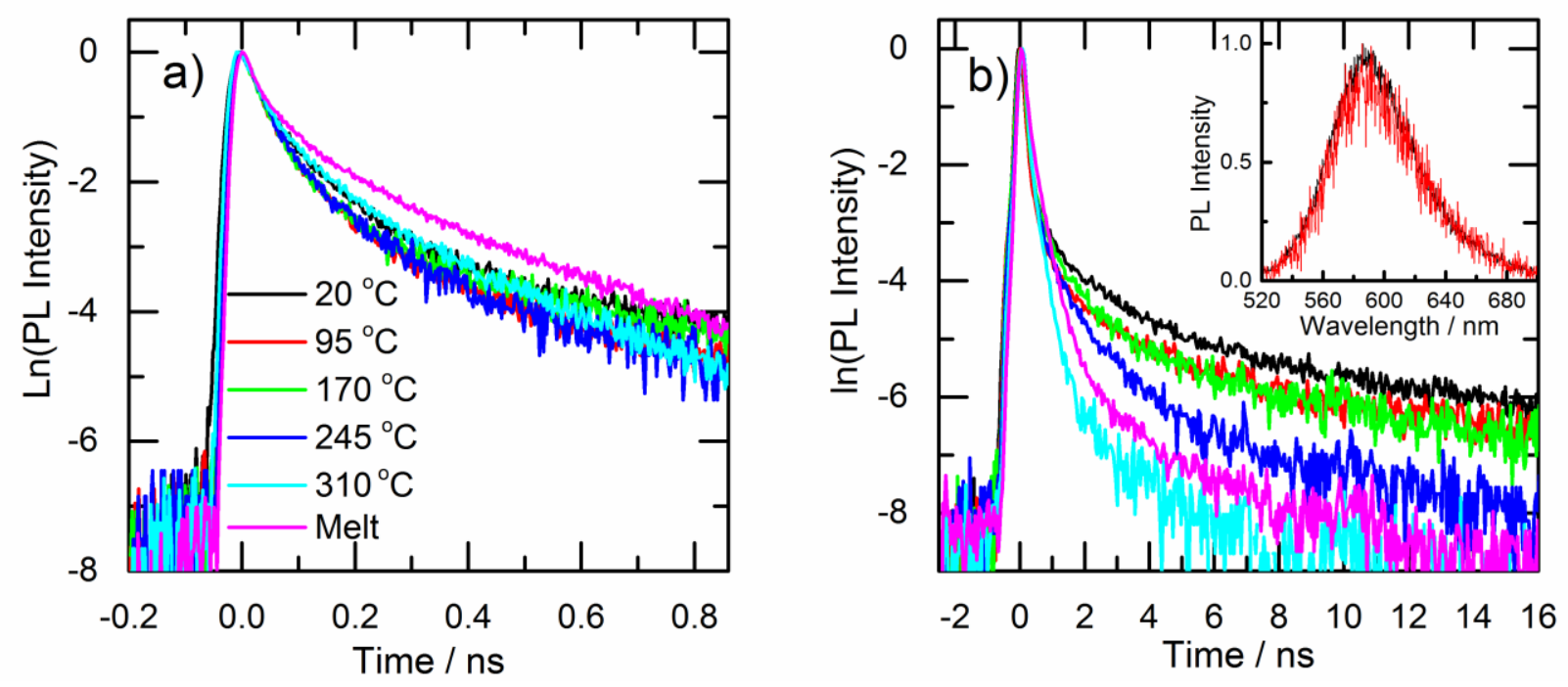

Figure 2. a) The prompt fluorescence decays $(0.0-0.9 \mathrm{~ns})$ of solid rubrene at different temperatures and the melt at $340{ }^{\circ} \mathrm{C}$. b) The delayed fluorescence decays $(0-16 \mathrm{~ns})$ of solid rubrene at different temperatures and the melt at $340{ }^{\circ} \mathrm{C}$. Inset: The fluorescence spectra of the melt integrated over the time windows 0.0 to $0.5 \mathrm{~ns}$ (black) and 0.5 to $3.5 \mathrm{~ns}$ (red). 

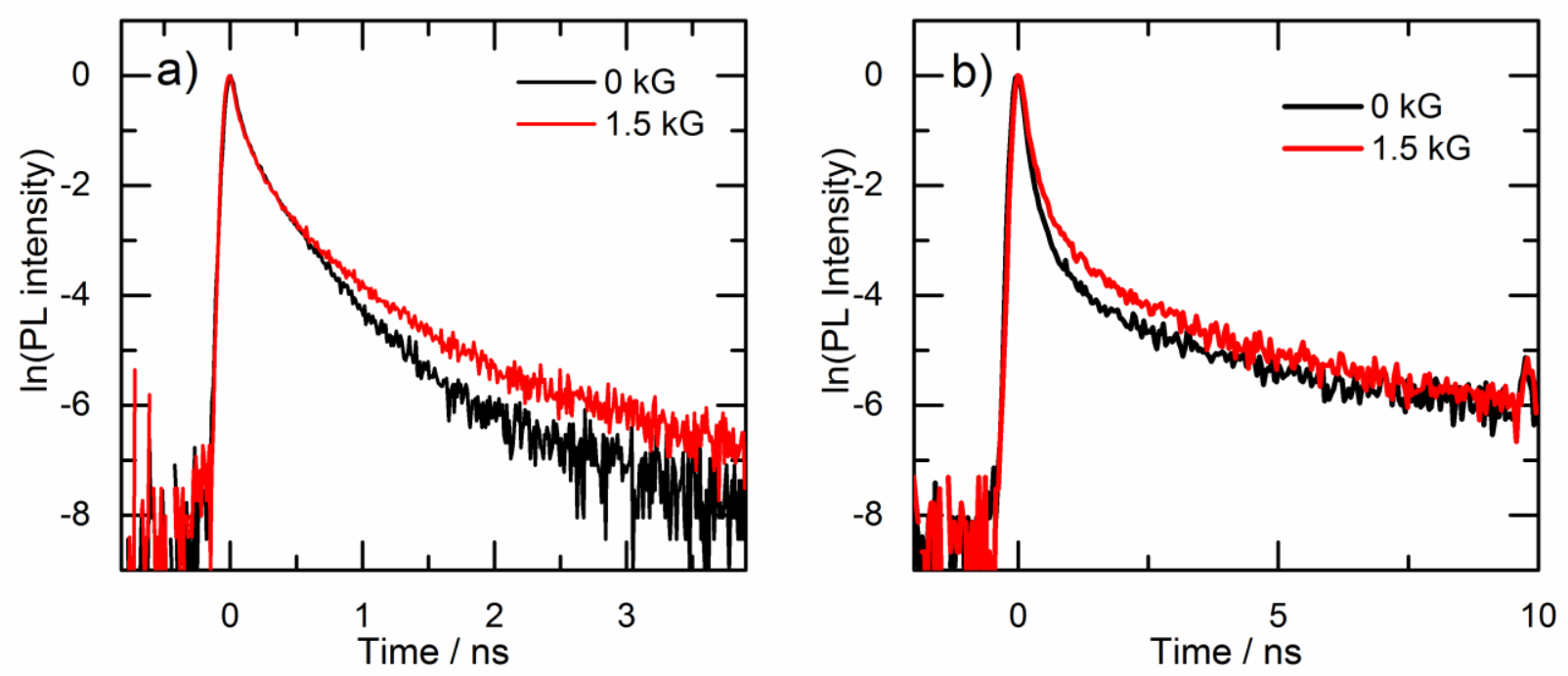

Figure 3. a) Time-resolved photoluminescence of the rubrene melt at $340{ }^{\circ} \mathrm{C}$ and b) solid rubrene powder at $20{ }^{\circ} \mathrm{C}$. Both samples shown an enhancement of the fluorescence signal at longer times with the application of a $1.5 \mathrm{kG}$ magnetic field. Note the different timescales of the two experiments due to the faster decay of the delayed fluorescence in the melt. 

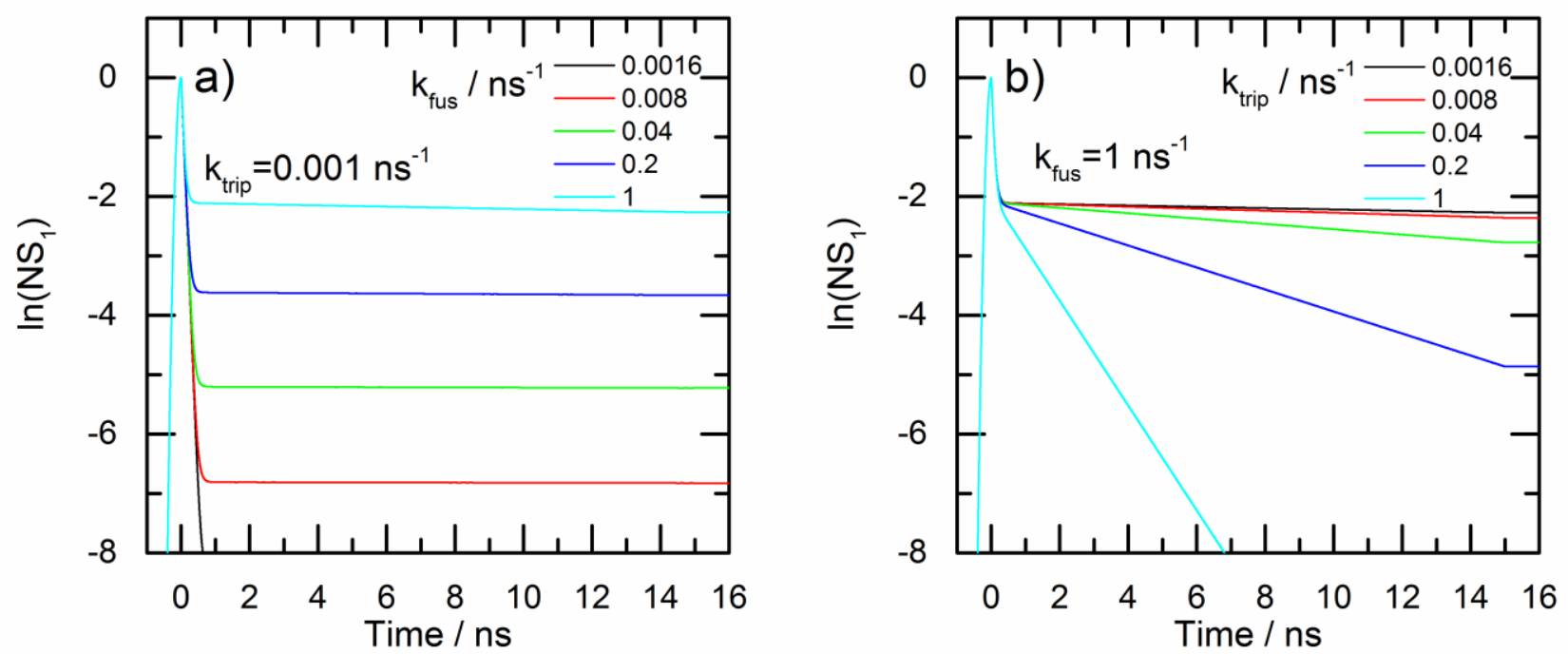

Figure 4. $N_{S I}(\mathrm{t})$ population decays (proportional to the observed photoluminescence signal) calculated using Equations (1) and (2). a) The triplet decay rate is set to $k_{t r i p}=0$ and the triplet fusion rate $k_{f u s}$ is varied. b) The triplet fusion rate is set to $k_{f u s}=1 \mathrm{~ns}^{-1}$ and the triplet decay rate $k_{\text {trip }}$ is varied. For all calculations, $\mathrm{k}_{\mathrm{rad}}=0.06 \mathrm{~ns}^{-1}$ and $\mathrm{k}_{\mathrm{fiss}}=15 \mathrm{~ns}^{-1}$. 


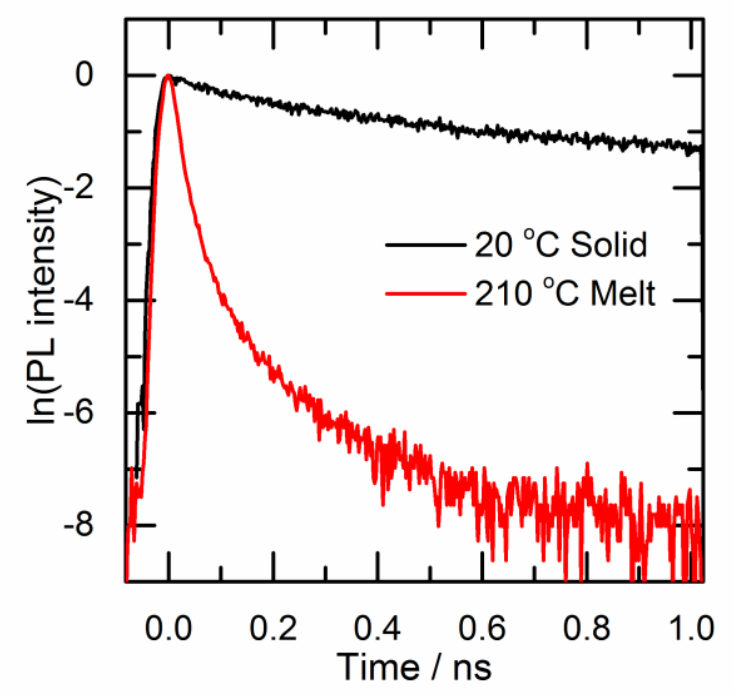

Figure 5. Photoluminescence decay of solid diphenylhexatriene at $20^{\circ} \mathrm{C}$ and the melt at $210^{\circ} \mathrm{C}$. The data have been integrated over the spectral window 425 to $550 \mathrm{~nm}$. 

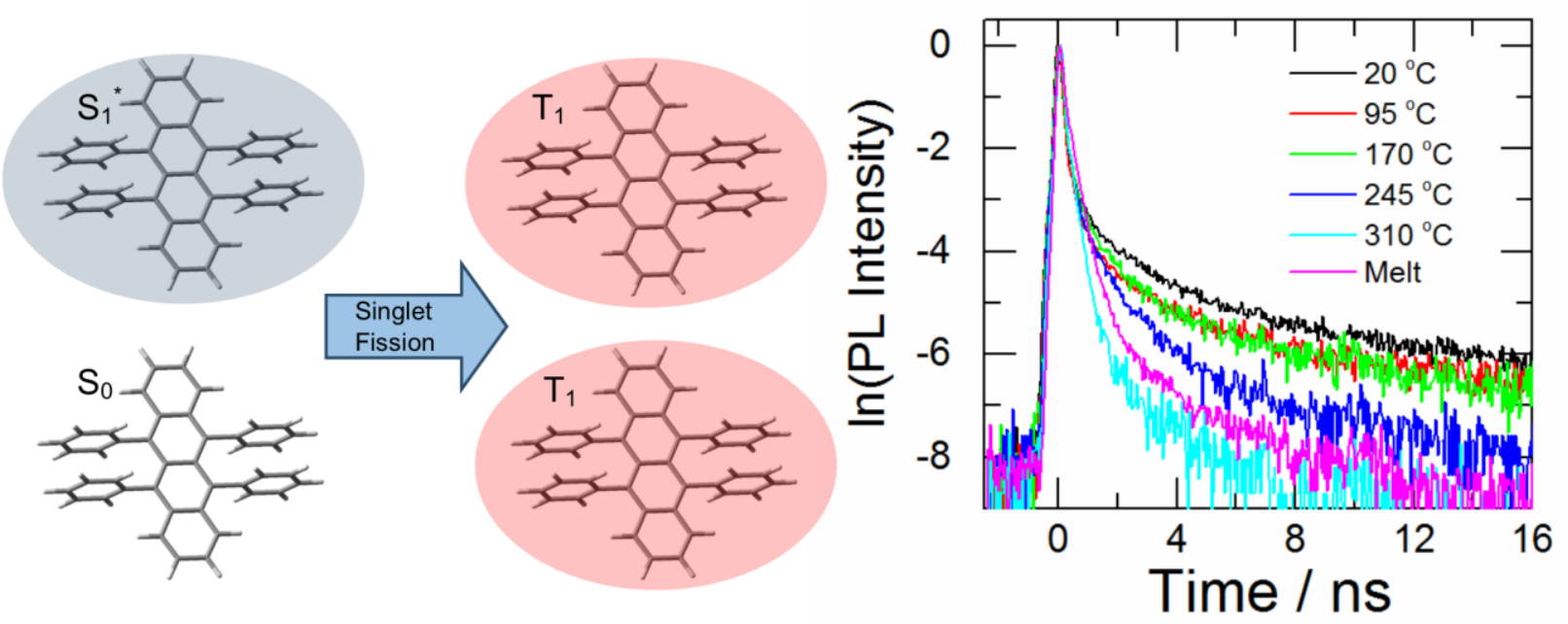